\title{
Der Handel als erstes Glied der Kette
}

\begin{abstract}
Als direkter Ansprechpartner der Konsumenten, ist der Handel auch für die ökologische Qualität der Produkte verantwortlich. Wie der Handel den damit verbundenen Anforderungen gerecht werden kann und welches die Voraussetzungen für ein erfolgreiches Stoffstrommanagement sind, darauf wird im folgenden Artikel eingegangen.
\end{abstract}

$\mathrm{D}$

Von Johannes Merck ie Anforderungen an die Produktqualität steigen. Dies betrifft klassische Qualitätsmerkmale wie technischen Standard, Haltbarkeit und Design, aber auch produktions- und humanökologische Aspekte und zunehmend solche der Sozialverträglichkeit. Diese Anforderungen werden von ganz unterschiedlichen Institutionen formuliert, vom Gesetzgeber, von Verbraucherschutzorganisationen, kirchlichen Hilfswerken, Naturschutzorganisationen und schließlich - vom Käufer. Zu erfiillen hat die Anforderungen in jedem Fall der Handel: Er ist der direkte Partner des Konsumenten im Wirtschaftsprozeß, er wird vor allem identifiziert mit der Qualität der von ihm angebotenen Produkte. Der Handel ist aus der Sicht des Anspruchstellers die relevante Bezugsgröße - er ist das erste Glied in der Kette.

Die Otto-Versand Handelsgruppe ist mit 35 Versandhandelsgesellschaften in 16 Staaten auf drei Kontinenten und einem Umsatz von rund 25 Milliarden Mark die größte Versandhandelsgruppe der Welt. Umweltschutz ist ein ausdrückliches Unternehmensziel der deutschen Muttergesellschaft. In Form einer firmeninternen Umweltpolitik hat sich das Unternehmen verpflichtet, einen kontinuierlichen Verbesserungsprozeß in Fragen der Umwelt sicherzustellen. Hierfür wurde ein Umweltmanagementsystem installiert, das sich an der EG-Umwelt-Audit-Verordnung orientiert. Der ökologische Optimierungsprozeß umfaßt alle Funktionsbereiche mit Umweltauswirkungen, neben dem Standort die Bereiche Transport und Verkehr, Verpackung sowie Werbemittel. Der Schwerpunkt der Optimierungsarbeit liegt auf der ökologischen Verbesserung des Sortiments.

Der Otto-Versand vertreibt 9.000 verschiedene Produkte - ein Vollsortiment. Über 50 Prozent dieser Produkte stammen aus dem Ausland, überwiegend aus Fernost. Was wissen wir von diesen Produkten? In der Regel nicht viel! Wir wissen, daß sie bestimmten Qualitätsanforderungen gerecht werden, daß Haushaltsgeräte bestimmte Energie- und Wasserverbrauchswerte nicht überschreiten, daß bestimmte Werkstoffe, z.B. Tropenholz, nicht verwendet werden. Viel umfangreicher als das, was wir wissen, ist das, was wir nicht wissen: Welche Materialien verarbeitet, welche Zusatzstoffe eingebracht wurden, wie der Rohstoff gewonnen wurde, welche Energie- und Wasserverbräuche in der Produktion entstanden, kurz: welche ökologische Relevanz ein Produkt hat?

Dieses Nichtwissen des Handels steht in einem wachsenden Widerspruch zu der Verantwortung für die Produktqualität, die er zunehmend trägt. Kann diesem Mißstand mit den Mitteln des Stoffstrommanagements abgeholfen werden? Sollte der Handel in diese Richtung aktiv werden? Vor welche Probleme stellt ihn das; welche Chancen stecken in der Schließung von Wertschöpfungsketten für den Handel?

\section{Der erste Versuch}

Der Otto-Versand hat bereits im Jahr 1993 einen ersten Versuch unternommen, diese Fragen in einem Praxistest zu klären. Damit wurden zwei Ziele verfolgt: Erstens sollte ein hochinnovatives, ökologisch höchsten Ansprüchen genügendes Produkt entstehen, zweitens sollten durch die Bildung strategischer Allianzen Kenntnisse über die ökologische Relevanz eines einzelnen Artikels - einer Baumwolltextilie - entlang der gesamten Wertschöpfungskette gesammelt werden. Mit diesen Kenntnissen sollte anschließend der breite Optimierungsprozeß für das gesamte Baumwollsortiment gezielter vorangebracht werden.

Das Projekt wurde ein Fehlschlag. Die beiden Ziele paßten nicht zusammen. Der entscheidende Fehler war, daß der Faktor Ökologie gegenüber der Ökonomie zu stark in den Vordergrund gerückt wurde. Dies hatte verschiedene Folgewirkungen, die das Projekt schließlich zum Scheitern brachten.

Dem Faktor Ökologie folgend, wurde bei der Partnerauswahl vor allem das ökologische Innovationspotential fokussiert. Dadurch kam zwar ein "Who is Who" der Textil-Industrie zusammen, aber keine organische Kette. Einige der verbundenen Unternehmen hatten noch nie zusammengearbeitet. Auch war die Zahl der beteiligten Firmen relativ hoch. Neben dem Händler, dem Konfektionär, dem Stoffproduzenten und dem Spinner beteiligten sich auch ein Chemieunternehmen sowie ein Maschinenbauer. Jedes Unternehmen hatte seine eigenen Vorstellungen davon, was es mit dem Projekt erreichen wollte. Und so wurde zwar heftig über die Anforderungen an textiles Stoffmanagement diskutiert, ein gemeinsames Handeln kam jedoch nicht zustande. Zu verschieden waren die Vorschläge zu Kostenrechnung, Kommunikationsstrategien und anderem. Alle Interessen und Erwartungen der Projektpartner berïcksichtigend, wäre am Ende zwar ein ökologisch anspruchsvolles Produkt herausgekommen und das in einer Stückzahl, mit der die Weltnachfrage nach ökologisch optimierten Produkten hätte befriedigt werden können- allerdings mit einem Preis, zu dem man es kaum hätte absetzen können.

Gerade auch die ökologische Kompetenz mancher Partner in der Kette belastete den Projekterfolg. Statt zu einer optimalen Transparenz, der Erschließung von Synergien und der Absprache konkreter technischer Innovationen, führte dies eher zu Eigenbrötelei und Abschottung. Es bestand nur eine geringe Bereitschaft, einmal erworbenes Know-how preiszugeben und damit möglicherweise Wettbewerbsvorteile einzubüßen. Ein wesentlicher Nutzen für die angestrebte, breite Sortimentsoptimierung war damit stark in Frage gestellt. Die Kooperation wurde beendet.

\section{- Weichenstellungen für den zweiten Anlauf}

Nach diesem Fehlschlag waren wir klüger. In einem zweiten Anlauf wurden sieben wesentliche Weichenstellungen vorgenommen, um ein Stoffstrommanagement-Projekt mit dem gleichen Ziel - der ökologischen Optimierung einer Baumwolltextilie - zum Erfolg zu führen:

- Der Faktor Ökologie wurde in die betriebswirtschaftlichen Ziele eingeordnet. 
(c) 20I0 Authors; licensee IÖW and oekom verlag. This is an article distributed under the terms of the Creative Commons Attribution Non-Commercial No Derivates License (http://creativecommons.org/licenses/by-nc-nd/3.o/), which permits unrestricted use, distribution, and reproduction in any medium, provided the original work is properly cited. 